\title{
Size and spatial correlation of defective domains in yttrium-doped $\mathrm{CeO}_{2}$
}

\author{
Stefano Checchia, ${ }^{1}$ Marco Scavini, ${ }^{1,2}$ Mattia Allieta, ${ }^{1}$ Michela Brunelli, ${ }^{1}$ Claudio Ferrero, ${ }^{3}$ and \\ Mauro Coduri ${ }^{1,4, a)}$ \\ ${ }^{I}$ Dipartimento di Chimica, Università degli Studi di Milano, Via C. Golgi 19, I-20133 Milano, Italy \\ ${ }^{2}$ ISTM-CNR and INSTM Unit, Via C. Golgi 19, I-20133 Milano, Italy \\ ${ }^{3}$ ESRF-The European Synchrotron, 71 Avenue des Martyrs, CS40220, 38043 Grenoble Cedex 9, France \\ ${ }^{4}$ CNR-IENI Institute for Energetics and Interphases, C.so Promessi Sposi 29, 23900 Lecco, Italy
}

(Received 29 September 2014; accepted 20 February 2015)

\begin{abstract}
The size of dopant-rich nanodomains was assessed in four samples of $\mathrm{Ce}_{1-\mu} \mathrm{Y}_{\mu} \mathrm{O}_{2-\mu / 2}$ through systematic pair distribution function (PDF) refinements. Experimental $G(r)$ curves were fitted by different structural models with the aim of finding a description which balanced precise structure parameterization and reasonable number of parameters. The most reliable model was a single $\mathrm{Y}_{2} \mathrm{O}_{3}$-like phase, which best accommodated to the close relationship between the fluorite $\left(\mathrm{CeO}_{2}\right.$-like $)$ and $\mathrm{C}$-type $\left(\mathrm{Y}_{2} \mathrm{O}_{3}\right.$-like) structures. In this model, a refined cation coordinate, $x(\mathrm{M} 2)$, measured the relative occurrence in the $G(r)$ of the chemical environment of $\mathrm{Y}$ and Ce at any value of $r$. The $r$-value at which $x$ (M2) vanished, i.e. at which the refined C-type cell becomes a redundant, low-symmetry description of a fluorite cell, was assumed as the size of a C-type domain. Subtle features in $G(r)$ could be attributed to the fluorite or C-type phase up to $\sim 500 \AA$ thanks to the narrow instrumental resolution function of the ID31 beamline (now ID22) at the ESRF, which allows us to get high resolution PDF data. (C) 2015 International Centre for Diffraction Data. [doi:10.1017/S0885715615000135]
\end{abstract}

Key words: total scattering, pair distribution function, non-stoichiometric oxides, doped ceria, defect structure

\section{INTRODUCTION}

Cerium oxide is widely investigated for its capability of forming solid solutions in the whole composition range with oxides of rare earths, transition metals (Minervini et al., 1999; Foschini et al., 2001), and group III cations (Park et al., 2000; Gregori et al., 2011). Particularly sought for is doping effected by substituting $\mathrm{Ce}^{4+}$ with trivalent cations from sesquioxides, in order to introduce oxygen vacancies according to the equilibrium Eq. (1):

$$
\frac{1}{2} M_{2} O_{3} \stackrel{\mathrm{Ce} \mathrm{O}_{2}}{\longrightarrow} M_{\mathrm{Ce}}^{\prime}+\frac{1}{2} V_{\ddot{o}}+\frac{3}{2} O_{o}
$$

Oxide ion vacancies are wanted for those applications requiring ionic conductivity. Charge transport and, in turn, ionic conductivity have an inverse dependence on the activation energy needed by the vacancies for migrating in the lattice. To this point, the crystal environment around the vacancies is of primary importance. In case of extended clustering of defects, oxygen vacancies would be trapped nearby the dopant cations in an energetically favourable environment (Goodenough, 2003). High activation energy barrier would ensue, resulting in low ionic conductivity. Conversely, if vacancies were not associated but scattered in the $\mathrm{CeO}_{2}$ structure, instead, they would be relatively free to migrate, thus, promoting ionic conductivity.

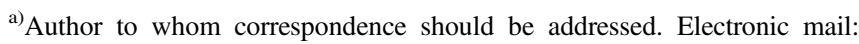
codurimauro@gmail.com
}

There is no agreement in the literature on how and even if the defects in doped ceria are aggregated in space. Conclusions are mainly biased by the techniques employed to investigate the defect structure. Bulk probes such as EXAFS and solid-state NMR (Yamazaki et al., 2000; Kim and Stebbins, 2007) suggest the formation of trimers $M_{\mathrm{Ce}}^{\prime}-V_{\ddot{o}}-M_{\mathrm{Ce}}^{\prime}$; however, such techniques only investigate the first few coordination shells of an atom. At odds with that, electron microscopy probes such as transmission electron microscopy (TEM), selected area electron diffraction (SAED), and electron energy loss spectroscopy (EELS) (Ou et al., 2006) suggest defect clustering up to a few nanometres. As a downside, the latter techniques only inspect a limited portion of the specimen, which may not be representative of the whole material.

To overcome some of those drawbacks, we recently exploited the pair distribution function (PDF) method to investigate doped ceria compounds upon doping with Gd (Scavini et al., 2012), Y (Coduri et al., 2012a, 2013a), and other rare earths (Coduri et al., 2012b, 2014), also under fuel cell operating conditions (Coduri et al., 2013b). The advantage of the PDF analysis over EXAFS lies in that the PDF function [i.e. $G(r)$ ] can yield information up to several hundreds of Angstroms in real space, depending on the instrumental resolution. The PDF technique is also bulk-probing, as it relies on the scattered intensity from all the active volume of the sample. These capabilities are well suited to studying doped ceria, which brings in the so-called nanostructure problem (Billinge and Levin, 2007): the structure of concern is singlephase though perturbed on average by large, doping-induced static disorder, as inferred from the compositional evolution 
of atomic displacement parameters (Coduri et al., 2012a, 2012b, 2013b; Scavini et al., 2012).

The key findings of the previous analyses cited above can be summarized as follows:

- The first coordination spheres in doped ceria samples can be pictured as the coexistence of dopant- and Ce-rich "droplets", i.e. sub-nanometre regions with a distorted fluorite $\left(\mathrm{CeO}_{2}\right)$ or $\mathrm{C}$-type (dopant oxide) structure. The relative amount of the two kinds of droplets follows the compound stoichiometry (Coduri et al., 2012a, 2012b, 2013b; Scavini et al., 2012).

- At intermediate dopant concentration $(0.25 \leq \mu<0.50)$, the dopant-rich droplets average out to C-type (dopant oxidelike) "domains", the size of which spans some hundreds of Ångstroms (Coduri et al., 2013a).

- C-type domains grow in size with increasing dopant concentration, and connect through antiphase domain boundaries (APDB). APDBs, appearing in the $Q$-space patterns as the selective line broadening of superstructure reflections (Coduri et al., 2013b), gradually fade with increasing dopant concentration.

In the frame of the investigation on doped ceria, this work specifically addresses the spatial correlation of defects by reviewing different structural models of the distribution of yttria-like defects in cerium oxide. The main structural features of $\mathrm{Ce}_{1-\mu} \mathrm{Y}_{\mu} \mathrm{O}_{2-\mu / 2}$ (CYO) are briefly recalled, pointing out the relationship between fluorite and C-type structures. In the latter, structural models are compared on the basis of their ability to fit to the experimental PDF curves for different compositions in ranges of interatomic distances spanning few hundreds of Ångstroms.

\section{EXPERIMENTAL}

\section{A. Synthesis of CYO}

Powdered samples of Y-doped ceria, CYO, were prepared according to the Pechini sol-gel method (Pechini, 1967), using $\mathrm{Ce}\left(\mathrm{NO}_{3}\right)_{3}$ and $\mathrm{Y}\left(\mathrm{NO}_{3}\right)_{3}$ (99 and 99.8\%, SigmaAldrich) in due proportions. The precursor powders from the hydrothermal synthesis were uniaxially pressed with a 7 ton load to $2 \mathrm{~mm}$-thick pellets, and sintered at $1173 \mathrm{~K}$ for $144 \mathrm{~h}$ with intermediate grindings. The obtained nominal compositions are $\mu=0,0.250,0.313,0.344,0.438,0.500$, and 1 .

\section{B. X-ray diffraction data collection, processing and analysis}

X-ray powder diffraction (XRPD) patterns were collected at the ID31 beamline (now ID22) of the ESRF, Grenoble. Isothermal measurements were carried out by setting $T=90 \mathrm{~K}$. The X-ray wavelength was $\lambda_{1}=0.35413$ (3) $\AA$, and data were collected up to $Q_{\max }=29.4 \AA^{-1}$, with $Q=4 \pi \sin \theta / \lambda$ the wavevector. The sample with $\mu=0.500$ was probed in a separate experimental run, using a wavelength $\lambda_{2}=0.3099(2) \AA$, reaching $Q_{\max }=28.0 \AA^{-1}$. The measured contribution of the empty capillary was subtracted from the PDF data.

The XRPD data were fitted using both the Rietveld method via the GSAS program (Larson and von Dreele, 2000) and its graphic interface EXPGUI (Toby, 2001), and the Whole Powder Pattern Modelling (WPPM) approach as implemented by the PM2K code (Leoni et al., 2006). $G(r)$ curves were obtained through the PDFGetX2 program (Qiu et al., 2004), and modelled with the PDFgui program (Farrow et al., 2007).

As a formalism, we used the so called reduced PDF, $G(r)$, obtained via the Fourier sine transform of the experimental total scattering function $S(Q)$ :

$$
\begin{aligned}
G(r)= & 4 \pi r\left[\rho(r)-\rho_{0}\right]= \\
& \frac{2}{\pi} \int_{0}^{\infty} Q[S(Q)-1] \sin (Q r) d Q
\end{aligned}
$$

where $\rho(r)$ is the atomic number density function, which denotes the probability of finding an atom at a distance $r$ from another atom, and $\rho_{0}$ is the average number density.

The $r$-dependent fits to the $G(r)$ curves were obtained following to the so-called "box-car" refinement strategy: selected structural parameters were iteratively refined throughout $20 \AA$ wide boxes covering a range up to $500 \AA$, depending on the model and the sample. Thus, the variable $r$ in the plots stands for the centroid of the "box-car" refinement range.

The degree of accuracy of the analysis was evaluated by calculating the residual factor as follows:

$$
R_{W}=\sqrt{\frac{\sum_{i=1}^{n} \omega\left(r_{i}\right)\left[G_{\mathrm{obs}}\left(r_{i}\right)-G_{\mathrm{calc}}\left(r_{i}\right)\right]^{2}}{\sum_{i=1}^{n} \omega\left(r_{i}\right) G_{\mathrm{obs}}^{2}\left(r_{i}\right)}}
$$

where $\omega\left(r_{i}\right)=\frac{1}{\sigma^{2}\left(r_{i}\right)}, \sigma\left(r_{i}\right)$ being the standard deviation for a given $r_{i}$ distance value.

\section{RESULTS AND DISCUSSION}

The fluorite structure of $\mathrm{CeO}_{2}$ is described by the space group $F m-3 m$ (225). In the $\mathrm{CeO}_{2}$ cell, $\mathrm{Ce}^{4+}$ occupies the $(0,0,0)$ position and has eight coordinated $\mathrm{O}^{2-}$ located in $(1 / 4,1 / 4,1 / 4)$. The $\mathrm{C}$-type structure of $\mathrm{Y}_{2} \mathrm{O}_{3}$, space group Ia-3 (206), can be seen as a $2 \times 2 \times 2$ fluorite supercell whose origin is shifted by $1 / 4,1 / 4,1 / 4$. In each octant, $\mathrm{Y}^{3+}$ occupies the sixfold coordinated $8 b$ and $24 d$ sites, $\mathrm{O}^{2-}$ is in the $48 e$ site, and two $16 c$ sites are vacant. Table I reports atom coordinates and Wyckoff positions of the pertinent phases. Hereafter, the C-type setting is applied to the fluorite structure, so that all sites are unambiguously referred to for any composition. We take the fractional $x$-coordinate of the M2 site [hereafter $x(\mathrm{M} 2)$ ] as the reference parameter to discuss our data, as it is the only degree of freedom of the cationic substructure: when $x(\mathrm{M} 2)=0$, the cation arrangement is the same as in fluorite, while it takes negative values in C-type.

TABLE I. Atom positions and corresponding Wyckoff symbols for the sites in fluorite, C-type, and fluorite with C-type setting. Notice that the $16 c$ site is vacant in pure $\mathrm{Y}_{2} \mathrm{O}_{3}$.

\begin{tabular}{llcl}
\hline \hline & \multicolumn{1}{c}{ Fluorite } & Fluorite with C-type setting & \multicolumn{1}{c}{ C-type } \\
\hline M1 & $4 a, 0,0,0$ & $8 b, 1 / 4,1 / 4,1 / 4$ & $8 b, 1 / 4,1 / 4,1 / 4$ \\
O1 & $8 c, 1 / 4,1 / 4,1 / 4$ & $48 e, 3 / 8,1 / 8,3 / 8$ & $48 e, x, y, z$ \\
M2 & & $24 d, 0,0,1 / 4$ & $24 d, x, 0,1 / 4$ \\
O2 & & $16 c, 3 / 8,3 / 8,3 / 8$ & $16 c, x, x, x$ \\
\hline \hline
\end{tabular}


TABLE II. Selected data from Rietveld refinements and WPPM best fits. For the $\mu=0.250$ sample the fluorite cell parameter $a=5.4032(5) \AA$ A has been doubled so as to report the value of the corresponding $2 \times 2 \times 2$ supercell. $U_{\text {mean }}$ is the msd parameter averaged over all the sites in each structure.

\begin{tabular}{lccccc}
\hline \hline$\mu$ & \multicolumn{1}{c}{0.250} & 0.3125 & 0.3438 & 0.4375 & 0.500 \\
\hline Phase & $\mathrm{F}$ & $\mathrm{C}$ & $\mathrm{C}$ & $\mathrm{C}$ & $\mathrm{C}$ \\
$a(\AA)$ & $10.8064(9)$ & $10.7917(9)$ & $10.7853(9)$ & $10.7738(9)$ & $10.759(7)$ \\
$x(\mathrm{M} 2)$ & 0 & $-0.0027(1)$ & $-0.00449(9)$ & $-0.01159(3)$ & $-0.01497(4)$ \\
$U_{\text {mean }}\left(\AA^{2}\right)$ & $0.00713(2)$ & $0.0104(4)$ & $0.0115(5)$ & $0.0137(8)$ & $0.0133(8)$ \\
$R_{\text {wp }}$ (Rietveld) & 0.0509 & 0.0795 & 0.0863 & 0.0652 & 0.0703 \\
APDB probability & & $0.12(2)$ & $0.080(4)$ & $0.06(3)$ & $0.0025(3)$ \\
$R_{\text {wp }}$ (WPPM) & & 0.0879 & 0.0899 & 0.0698 \\
\hline \hline
\end{tabular}

\section{A. Reciprocal space analysis}

Table II outlines the results from Rietveld refinements of the XRPD patterns. All of the CYO samples were singlephase, and the average structure is $F m-3 m$ fluorite for $\mu \leq$ 0.250 and Ia-3 C-type for $\mu>0.250$. The cell parameter decreases with increasing yttrium concentration owing to the lower coordination and smaller ionic radius of $\mathrm{Y}^{3+}$ with respect to $\mathrm{Ce}^{4+}$ (Shannon and Prewitt, 1969; Coduri et al., 2012b).

As depicted in Figure 1, O2 oxygen vacancies (16c) and $\mathrm{Ce} / \mathrm{Y}$ substitutional defects cause the $x$-coordinate of the C-type M2 cation to take intermediate values between those corresponding to the end members of the solid solution, that is $x(\mathrm{M} 2)=0$ for $\mathrm{CeO}_{2}$, and $x(\mathrm{M} 2) \sim(-0.032)$ for $\mathrm{Y}_{2} \mathrm{O}_{3}$. Such values are consistent with the distribution of $\mathrm{M} 2$ cations in two different chemical environments: (i) the centre of eight equidistant occupied oxygen sites $\left[\mathrm{CeO}_{2}\right.$-like cuboids, Figure 1(a)], and (ii) an offset position because of two vacant $\mathrm{O} 2$ sites $\left[\mathrm{Y}_{2} \mathrm{O}_{3}\right.$-like cuboids, Figure $\left.1(\mathrm{c})\right]$.

The C-type structure of the samples with $\mu=0.313,0.344$, and 0.438 is largely distorted with respect to reference $\mathrm{Y}_{2} \mathrm{O}_{3}$. The atomic mean square displacement (msd) peaks at $\mu=$ 0.438 (see Table II). Since measurements were performed at $90 \mathrm{~K}$, the trend in the msd parameters is because of static disorder only.

Also recalled in Table II are the results of the WPPM best fits to XRPD patterns of the $\mu=0.313,0.344$, and 0.438 samples, obtained using PM2K. Accurate fit of the superstructure peaks were attained only by accounting for antiphase domains with dopant-like structure. Ordering of defects starting at nonequivalent sites could have yielded unmerged domains splicing at APDBs. A similar mechanism was proposed for the $\mathrm{Cu}_{3} \mathrm{Au}$ alloy (Scardi and Leoni, 2005).

A possible relationship between the APDB probability and the spatial extent of such domains prompted us to employ PDF analysis in order to measure the domain correlation distance.

\section{B. Real-space analysis}

The effect of composition on the cation environment is witnessed by the metal-metal (MM) nearest neighbour distances in Figure 2. At the lowest dopant concentration already, the single MM distance of $\mathrm{CeO}_{2}$ [Figure 2(a)] splits into a shorter (MMa 3.75 $\AA$ ) and a longer distance (MMb 4.10 $\AA)$. The $G(r)$ of all the samples was fitted in the range between 1.5 and $5 \AA$. With reference to $\mu=0.250,0.344$, and 0.500 samples, Figures 2(b)-2(d) show the $G(r)$ in the $3<r<5 \AA$ range as fitted by fluorite $(\mu=0.250)$ and C-type $(\mu=0.344$ and 0.500$)$ long-range models. As to the $\mu=0.250$ sample, a fluorite phase could not reproduce the MMb peak. In fact, the $\mathrm{MMb}$ interatomic distance tallies with an atom pair involving the C-type $\mathrm{M} 2$ site [see $\mathrm{Y}_{2} \mathrm{O}_{3}$ in Figure 2(h)]. However, in the $G(r)$ of the $\mu=0.344$ and 0.500 samples, the MMb peak is equally mismatched when a C-type phase is fitted into it.

Either single phase fitted poorly the $G(r)$ in this range, as neither of them could be adapted to fit $G(r)$ peaks originating from the other phase. A reasonable solution was provided by a (a)

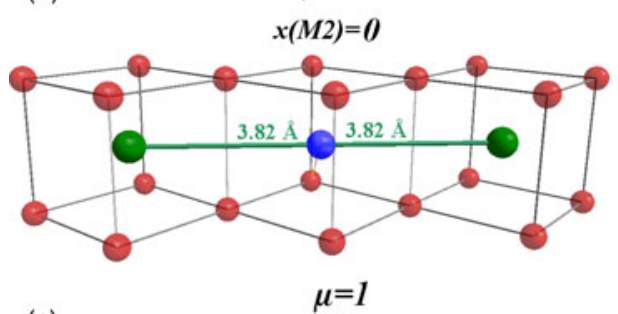

(c)

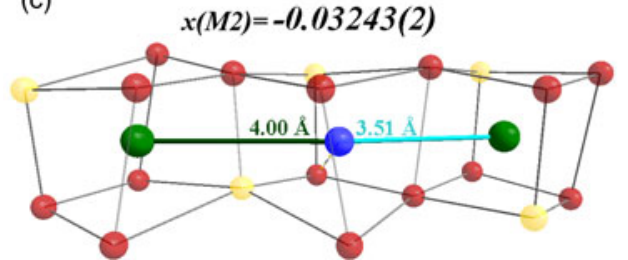

(b)
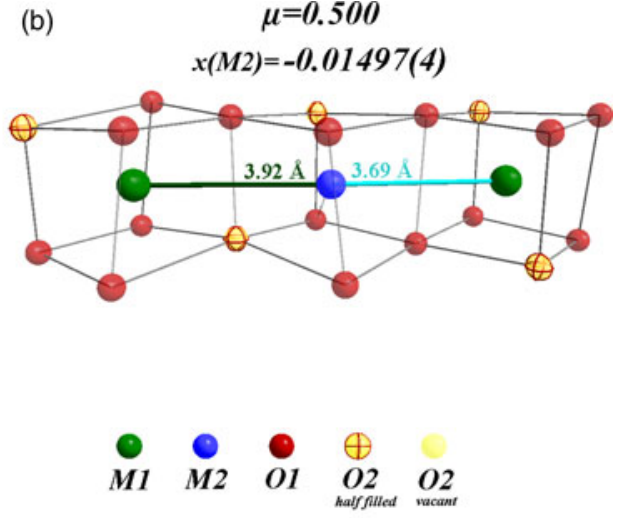

Figure 1. (Colour online) (a) $\mathrm{CeO}_{2}$ cuboid, where the $\mathrm{Ce}^{4+}$ cation $\left(\mathrm{M} 1=\mathrm{M} 2\right.$ ) is centred in the eight-coordinated position; (b) $\mathrm{Ce}_{0.5} \mathrm{Y}_{0.5} \mathrm{O}_{1.75}$ cuboid, with 0.5 probability for a vacancy to be in the $16 c$ site and a displaced M2 cation; (c) $\mathrm{Y}_{2} \mathrm{O}_{3}$ cuboid, in which every $16 c$ site is empty. For each panel $x$ (M2) yielded by the respective Rietveld fit is indicated. 


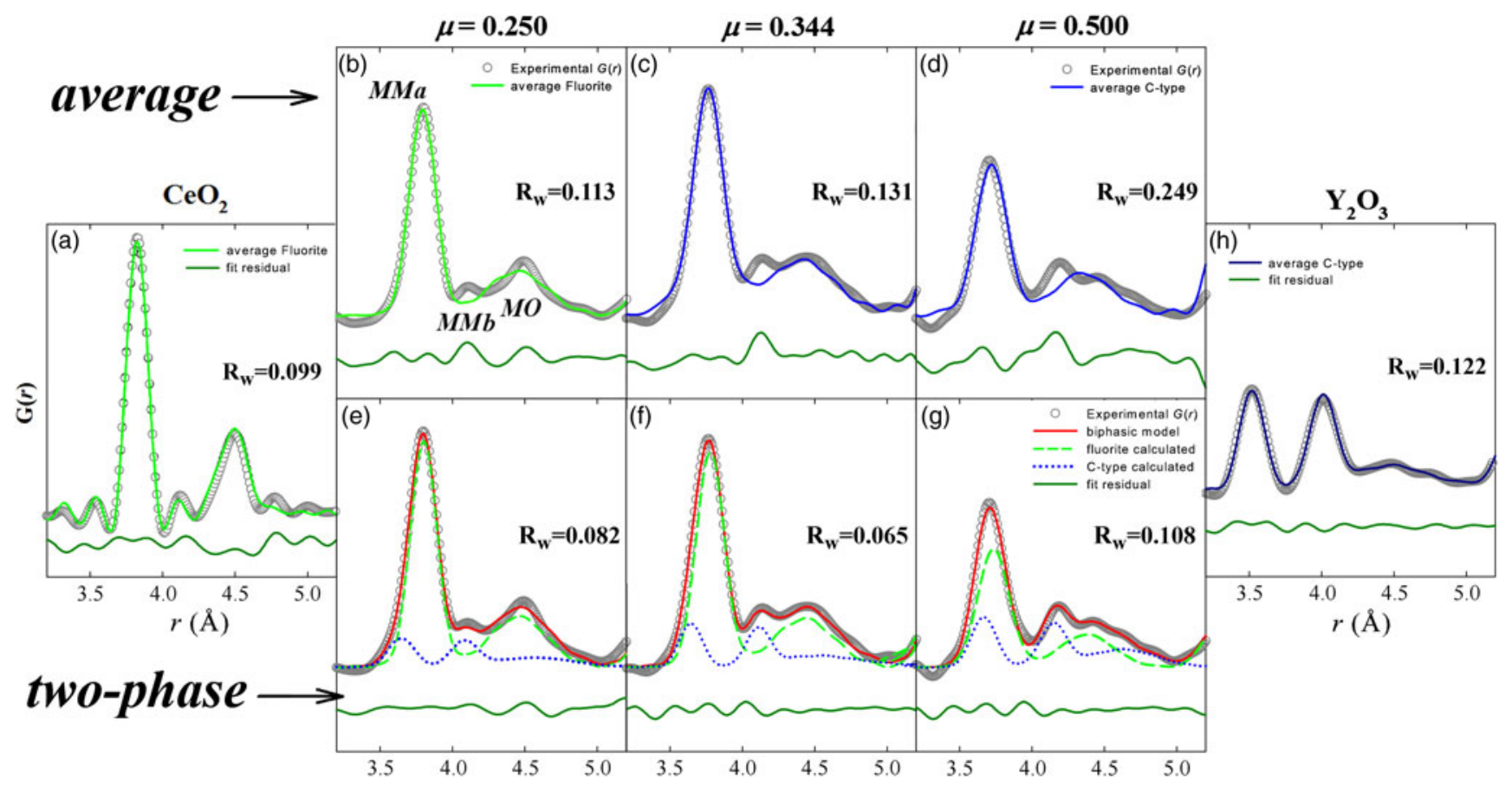

Figure 2. (Colour online) Experimental (circles) PDF curves in the $3<r<5$ A range for samples with $\mu=0.250,0.344$, and 0.500 composition fitted above (b-d) by the fluorite (green dashed line) and C-type (blue solid line) average models; below (e-g) by the two-phase model (red solid line), to which the partial contributions of the fluorite (green solid line) and C-type (blue dotted line) phases are also plotted. (a) Best fit based on the single-phase fluorite structure for $\mathrm{CeO}_{2}$ (solid line); (h) best fit based on the single-phase C-type structure for $\mathrm{Y}_{2} \mathrm{O}_{3}$ (blue solid line). Fit residuals are indicated in each frame (solid line below the fitted curves and right-hand side of the frame).

two-phase model, which considers the first atomic coordination shells as a combination of the fluorite and C-type chemical environments (Coduri et al., 2012b; Scavini et al., 2012). The fit by the two-phase model of the $G(r)$ curves is depicted in Figures 2(e)-2(g) together with the partial contributions from the fluorite and C-type phases. An overview of the refined parameters is given in Table III (see "relaxed twophase"). The change in the relative intensity and position of the MMa and MMb peaks upon increasing $\mu$, i.e. the lesser occurrence of pairs involving Ce replaced by $\mathrm{Y}-\mathrm{M}$ pairs is accounted for not only by the phase scale-factor parameters, but also by $x$ (M2). At $\mu=0.250$ already, the value of $x$ (M2) refined by the two-phase model is close to that in $\mathrm{Y}_{2} \mathrm{O}_{3}$ despite the fluorite average structure of this sample.
This limited spatial extent over which C-type and fluorite interatomic distances are described by the two-phase model was defined as "droplet" (Scavini et al., 2012; Coduri et al., 2013a). As a result of both the tiny coherence length and random orientation, droplets average out to zero in the reciprocal space and do not constitute a secondary phase in the XRPD patterns. Thus, using a two-phase model for describing the first few atomic distances is consistent with the observation in the diffraction patterns that every CYO sample is single-phase.

To find out how the dopant-like atomic environment relaxes out of the first coordination shell, C-type and fluorite contributions must be probed further in the $r$ space for each sample. High-quality PDF data were needed to apply different models to the experimental $G(r)$ on a wide $r$ range. Figure 3(a) reports

TABLE III. Overview of the structural assessed in this work with the respective box-car refinement parameters. Scale (f,c) stands for the relative phase fractions; $a_{\mathrm{F}}$ and $a_{\mathrm{C}}$ are the cell parameter of fluorite and C-type phases, respectively; $U_{\text {iso }}$ indicates the isotropic msd (when $U_{\text {iso }}$ is applied to two atoms, both share the same value); $U_{i i}$ is an anisotropic component of msd.

\begin{tabular}{|c|c|c|c|c|c|}
\hline Model & Unrelaxed two-phase & Relaxed two-phase & C-type isotropic & C-type anisotropic & Fluorite \\
\hline $\begin{array}{l}\text { Number of parameters } \\
\text { Refined parameters }\end{array}$ & $\begin{array}{l}4 \\
\text { Overall scale } \\
\text { Scale (f,c) } \\
U_{\text {iso }}(\mathrm{M} 1-\mathrm{M} 2) \\
U_{\text {iso }}(\mathrm{O} 1)\end{array}$ & $\begin{array}{l}7 \\
\text { Overall scale } \\
\text { Scale }(\mathrm{f}, \mathrm{c}) \\
x(\mathrm{M} 2) \\
a_{\mathrm{F}} \\
a_{\mathrm{C}} \\
U_{\text {iso }}(\mathrm{M} 1, \mathrm{M} 2) \\
U_{\text {iso }}(\mathrm{O} 1)\end{array}$ & $\begin{array}{l}6 \\
\text { Overall scale } \\
a_{\mathrm{C}} \\
x(\mathrm{M} 2) \\
U_{\text {iso }}(\mathrm{M} 1) \\
U_{\text {iso }}(\mathrm{M} 2) \\
U_{\text {iso }}(\mathrm{O} 1, \mathrm{O} 2)\end{array}$ & $\begin{array}{l}10 \\
\text { Overall scale } \\
a_{\mathrm{C}} \\
x(\mathrm{M} 2) \\
U_{x x}(\mathrm{M} 1) \\
U_{x y}(\mathrm{M} 1) \\
U_{11}(\mathrm{M} 2) \\
U_{22}(\mathrm{M} 2) \\
U_{33}(\mathrm{M} 2) \\
U_{23}(\mathrm{M} 2) \\
U_{\text {iso }}(\mathrm{O} 1, \mathrm{O} 2)\end{array}$ & $\begin{array}{l}4 \\
\text { Overall scale } \\
a_{\mathrm{F}} \\
U_{\text {iso }}(\mathrm{M}) \\
U_{\text {iso }}(\mathrm{O})\end{array}$ \\
\hline
\end{tabular}



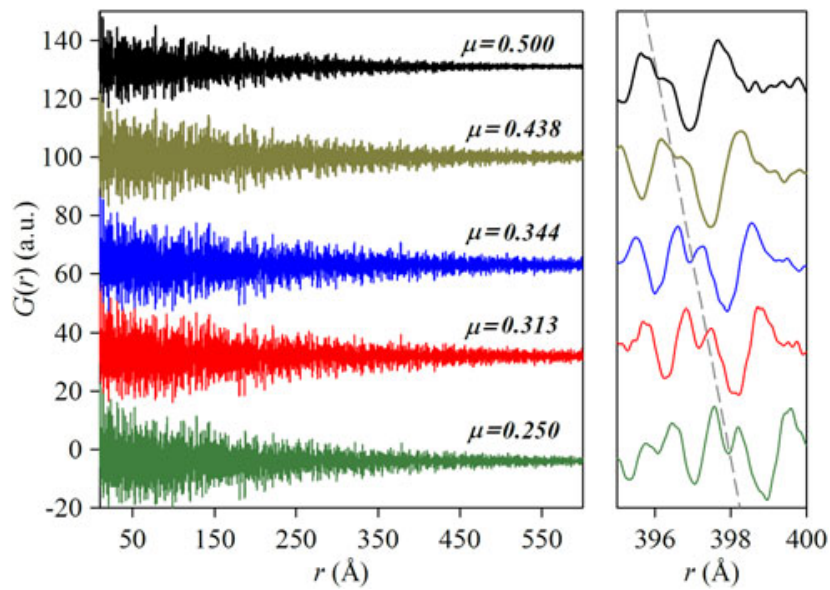

Figure 3. (Colour online) Experimental $G(r)$ curves up to $r=600 \AA$ (left) for the CYO samples. Details of the curves in the range $395<r<400 \AA$ are also shown (right). The dashed line is a guide to the eye tracing the peak position.

the $G(r)$ up to $600 \AA$ for the $\mu=0.250,0.313,0.344,0.438$, and 0.500 samples. The slow falloff of the $G(r)$ amplitude owes to the narrow instrumental resolution function of the ID31 diffractometer. Noise in the PDF data was minimized by increasing counting statistic thanks to: (i) long collection time, especially in the high $Q$ range, (ii) low temperature of the experiment $(90 \mathrm{~K})$, which kept the Debye-Waller factor low.

In the $G(r)$ curves discussed, signal-to-noise ratio and peak resolution were satisfactory even at $400 \AA$ A. For instance, we could detect a shift in position, shape, and width of the two peaks of the doublet at $\sim 397 \AA$ depicted in Figure 3(b). The traces of this doublet upon increasing $\mu$ match the shortening of the interatomic distances and the increase in the DW factor at $\mu=0.438$ worked out in reciprocal space.

In the following paragraphs, structural parameters refined against $G(r)$ in successive $20 \AA$ ranges (box-car refinements) are discussed through their trends as a function of $r$. A list of the refined parameters for the models introduced is given in Table III.

As it yielded the best solution on the local scale, the twophase model was tested on further interatomic distances in two different ways: (i) by keeping the positional degrees of freedom [cell parameters and $x(\mathrm{M} 2)]$ fixed, as in the local scale $(r<5 \AA)$ refinement, referred to as "unrelaxed two-phase"; (ii) by refining the positional degrees of freedom, referred to as "relaxed two-phase". Hereafter we will call the refined scale-factor of the C-type phase as " $C$-fraction".

Figure 4(a) shows the unrelaxed two-phase model fitted against the $G(r)$ of the $\mu=0.250$ sample in the $5<r<15 \AA$ range. It can be noted from the misshapen peaks and the high fit residual that such model becomes unreliable as soon as the fluorite and C-type distances are not resolved. Actually, for $r>10 \AA$, the $G(r)$ vectors span over the length of a droplet, and thereby they cannot be decomposed into the distinct contributions from two different coordination environments. By letting also phase cell parameters and $x(\mathrm{M} 2)$ be refined, the fit quality improved significantly [Figure 4 (b)], hence, the $r$-dependent structure refinement was carried up to hundreds of $\AA$ out using the relaxed model.

The trends of $x$ (M2) and of $C$-fraction as a function of $r$ obtained using the relaxed two-phase model are plotted in Figures 5(a) and 5(b). The cell parameters of the two phases

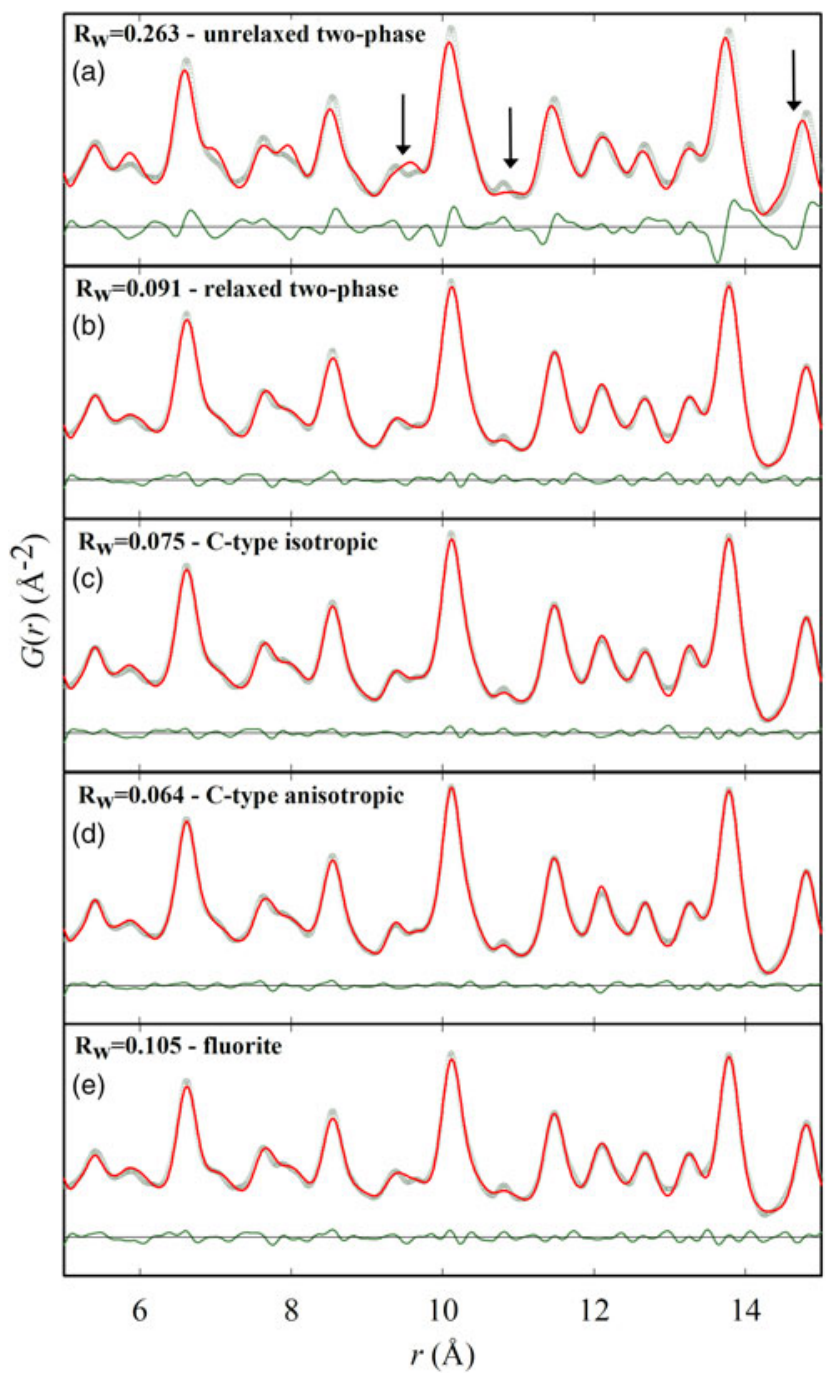

Figure 4. (Colour online) Experimental (circles) and model fitted PDF curves in the $5<r<15 \AA$ range for the sample with $\mu=0.250$ composition: (a) unrelaxed two-phase model; (b) relaxed two-phase model; (c) isotropic C-type model; (d) anisotropic C-type model; (e) fluorite model. Below each fitted curve the fit residual (green solid line) is shown along with its zero-point line (dotted line).

correlate strongly when refined. Notably, $C$-fraction for the $\mu=0.250$ sample vanishes just over $100 \AA$, while the corresponding $x(\mathrm{M} 2)$ value becomes unphysically negative. A similar observation can be made for the $\mu=0.344$ sample. In particular, in this case, the $C$-fraction cut-off is shifted to a higher $r$ value $(\sim 210 \AA)$, but it is poorly determined as its value declines to zero very slowly. Unlike this trend, in the sample $\mu=0.313, x(\mathrm{M} 2)$ goes neatly to zero, while $C$-fraction is almost constant with $r$.

Despite the large fluctuation in the values because of high parameter correlation, such trend suggests a structural evolution from C-type to fluorite when increasing $r$ in the samples $\mu=0.250,0.313$, and 0.344 . Indeed, the vanishing of either the $C$-fraction or $x(\mathrm{M} 2)$ supports the only presence of the fluorite phase at high $r$. On the other hand, the $\mu=0.438$ sample shows no fluorite character even at $500 \AA$.

Bearing in mind that both the $x(\mathrm{M} 2)$ and $C$-fraction parameters express the deviation from the fluorite structure, and that the two parameters exhibit a major negative correlation, we derived a C-type-to-fluorite order parameter by multiplying 
$x$ (M2) by $C$-fraction through the whole refinement range. The $x$ (M2) $\times C$-fraction parameter, plotted in Figure 5(c), becomes zero at $r \sim 120, \sim 110$, and $\sim 200 \AA$, respectively, for $\mu=0.250$, 0.313 , and 0.344. As expected, at $\mu=0.438$, the $x(\mathrm{M} 2) \times$ $C$-fraction parameter does not approach zero at any distance.

In the refinements against the relaxed two-phase model, the C-type cell parameter converged to a value twice the fluorite cell parameter. Therefore, a meaningful description of the structure could be achieved using a single C-type model [Figure 4(c)], thereby cutting the correlation between fluorite and C-type structural parameters. In the C-type model, site occupancy factors of $\mathrm{Ce}$ and $\mathrm{Y}$ atoms mirror the sample composition, and the $16 c$ site is filled accordingly. As a result, the $x$ (M2) parameter returned by the refinement was averaged over the two unalike positions of $\mathrm{Ce}$ and $\mathrm{Y}$ in their chemical environment, thus including the relative weights of the fluorite and C-type phases. While this was not satisfying on the local scale, it afforded a good fit at the nanometre scale.

Lower fit residual was achieved as compared with the relaxed two-phase model. The cell parameters evolve negligibly

(a)

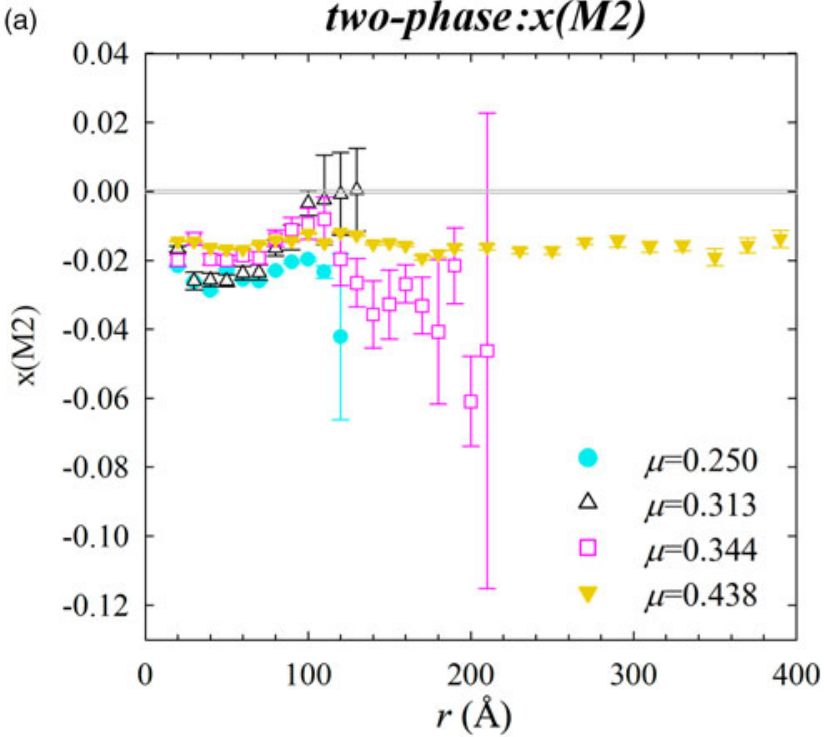

(c) two-phase:x(M2)*C-fraction

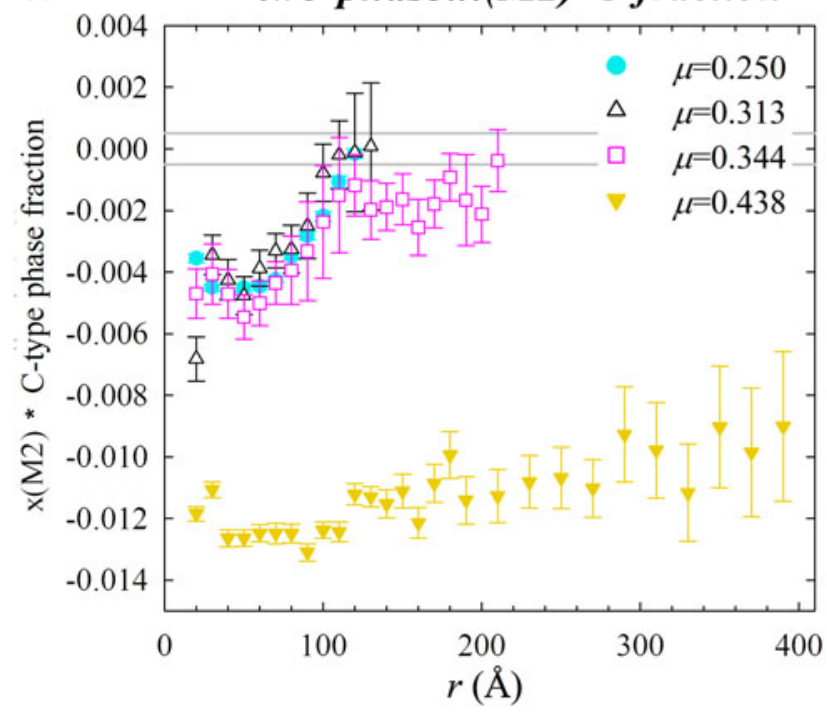

along $r$ in all of the samples, and closely resemble the respective values from Rietveld refinements: $a \sim 10.807, ~ 10.787$, $\sim 10.780$, and $\sim 10.769 \AA$ for $\mu=0.250,0.313,0.344$, and 0.438 . No noteworthy correlations involving $x(\mathrm{M} 2)$ were found and, therefore, the C-type domain size could be accurately estimated by only using the $r$-dependence of $x(\mathrm{M} 2)$.

The trends of $x(\mathrm{M} 2)$ vs. $r$ yielded by the C-type model are plotted in Figure 5(d). For $\mu=0.250,0.313$, and 0.344, $x$ (M2) averages out to zero at $r \sim 120, \sim 140$, and $\sim 180 \AA$, respectively. On the contrary, $\mu=0.438, x(\mathrm{M} 2)$ slopes in the range $-0.014<$ $x(\mathrm{M} 2)<-0.010$, but does not become zero within a range of $500 \AA$. This result points out that the C-type phase virtually achieves long-range coherence at $\mu=0.438$, whereas the same ordering is confined in 100-200 А-sized domains at lower dopant concentrations. As can be noticed in Figure 5(d), the value of $x$ (M2) within the domain gets more negative the higher the $Y$ concentration in the sample. With increasing $\mu$, fewer fluoritelike interatomic distances in the $G(r)$ curve must be fitted by the C-type phase. Accordingly, the refined $x(\mathrm{M} 2)$ parameter resembles more closely the value of $x(\mathrm{M} 2)$ in $\mathrm{Y}_{2} \mathrm{O}_{3}$.
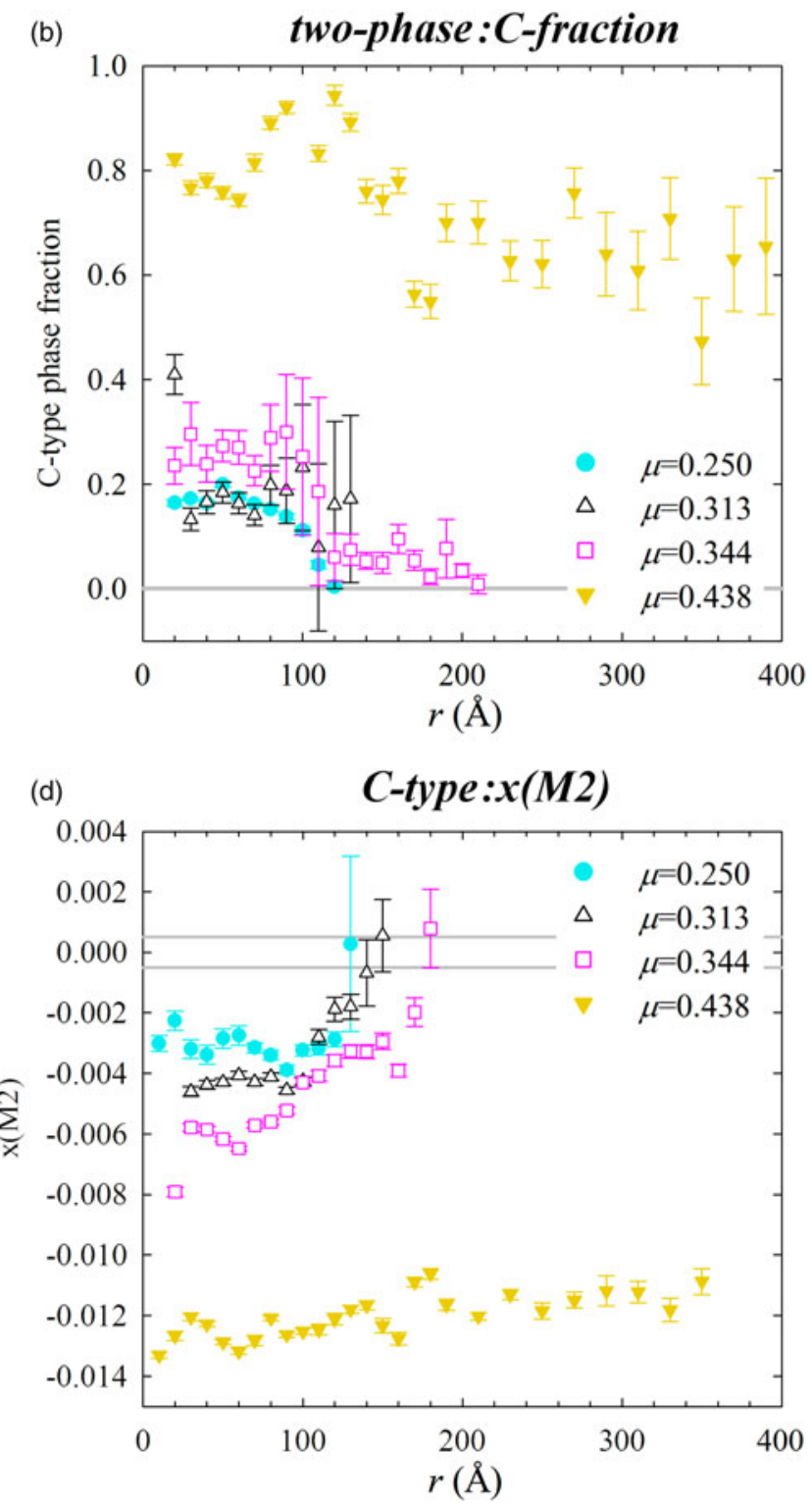

Figure 5. (Colour online) Plots of the refined parameters vs. $r$ for the samples $\mu=0.250,0.313,0.344$, and 0.438 as from: (a-c) the relaxed two-phase model, (d) the isotropic C-type model. Horizontal solid lines in the plots indicate $x(\mathrm{M} 2) \pm 0.004$ as a reference. 


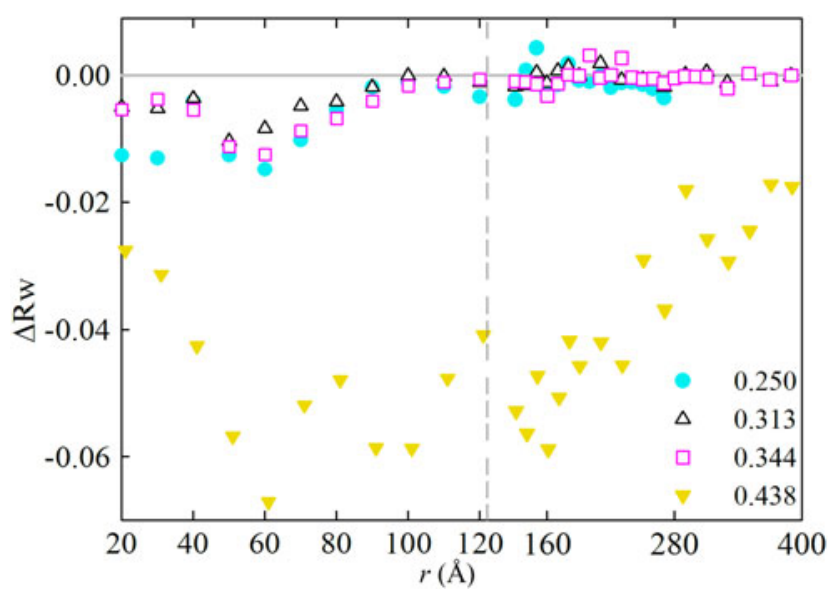

Figure 6. (Colour online) Plot of the difference, $\Delta R_{w}$, between the fit residual as from the C-type isotropic model and from the fluorite model as a function of $r$ for the samples $\mu=0.250,0.313,0.344$, and 0.438 . On the left of the dashed vertical line the $r$-axis scale is expanded.
A possible improvement of the fitting model could be achieved by using a C-type phase along with anisotropic displacement parameters. Strong anisotropic static disorder is indeed typical of doped ceria compounds (Scavini et al., 2012; Coduri et al., 2013b). Figure 4(d) shows the $G(r)$ fit by this model in the $5<r<15 \AA$ range for the $\mu=0.250$ composition.

Lower fit residual with respect to the previous C-type model (i.e., isotropic C-type) was attained using anisotropic thermal factors (i.e., anisotropic C-type). The refined cell parameter matched the value obtained by the isotropic C-type model for any composition and $r$ range.

For the $\mu=0.344$ and 0.438 samples, the $r$-dependence of $x$ (M2) parameter followed almost the same trend yielded by the isotropic model. Conversely, for the $\mu=0.250$ and 0.313 samples a lower $x(\mathrm{M} 2)$ cut-off resulted $(\sim 40 \AA)$ which totally disagrees with isotropic model results. This discrepancy is explained by the strong correlation between the $x(\mathrm{M} 2)$ position and the $U_{11}$ parameter, which made this model inconsistent. Using the anisotropic C-type, the uncertainties on $x(\mathrm{M} 2)$

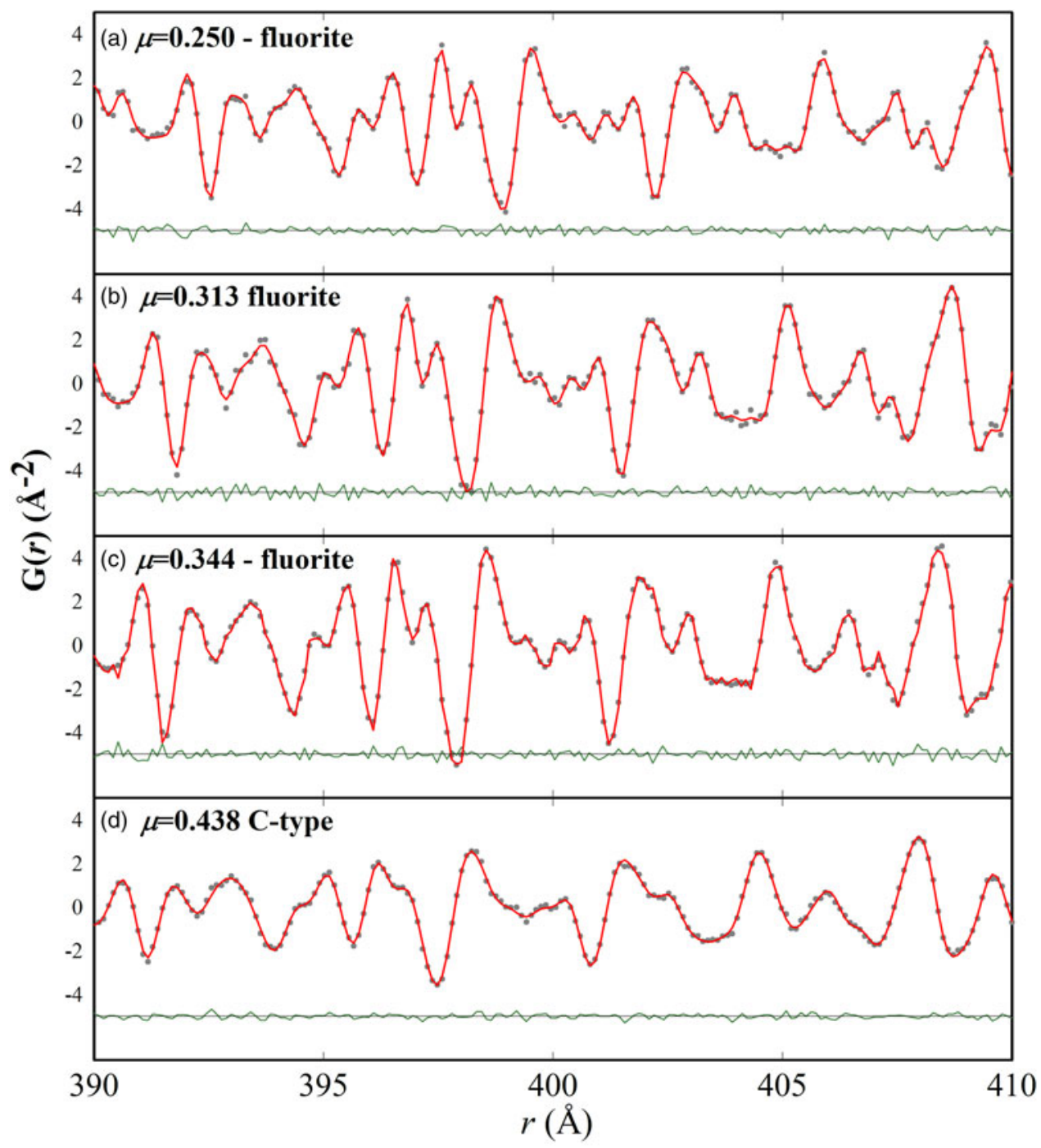

Figure 7. (Colour online) Experimental (circles) PDF curves in the $390<r<410 \AA$ range for the samples (a) $\mu=0.250$, (b) $\mu=0.313$, (c) $\mu=0.344$, and (d) $\mu=0.438$ with the corresponding calculated profile (solid red line) based on the respective best fitting model. Below each fitted curve the fit residual (solid green line) is shown along with its zero-point line (dotted line). 
were greater by an order of magnitude than those found using the isotropic C-type. For these reasons, we retained the isotropic C-type as the reference model for estimating domain size.

By inspecting the refined C-type phase of the samples $\mu=$ $0.250,0.313$, and 0.344 , it must be pointed out that the uncertainty on $x$ (M2) increased considerably as $x(\mathrm{M} 2)$ approaches zero. Such an increase suggests that the $G(r)$ might be fitted more accurately in the $r$ range encompassing the domain boundary and further in $r$. Thus, a simple fluorite structural model was fitted against the $G(r)$ up to $400 \AA$, so that the refined C-type and fluorite phases could be compared throughout $r$.

The difference, $\Delta R_{w}\left[=R_{w}(\mathrm{C}\right.$-type $)-R_{w}($ fluorite $\left.)\right]$, between the residual of fit by $\mathrm{C}$-type and that by fluorite is plotted in Figure 6 for the four compositions $\mu=0.250,0.313$, 0.344 , and 0.438 . The $\Delta R_{w}$ difference is negative in the samples $\mu=0.250,0.313$, and 0.344 within the respective domain size, indicating the better agreement of the C-type model with the observed $G(r)$. The two fit residuals cancel each other out approximately at the domain boundary, then, $\Delta R_{w}$ oscillates around zero in the further range of $r$, at which the refined phases virtually equate to each other. In fact, when a C-type phase was fitted against $G(r)$ in an $r$ range outside the C-type domain, $x(\mathrm{M} 2)$ equalled 0 within the uncertainty. In the $\mu=0.438$ sample, the fit residuals never cross in the whole range of $r$, reflecting the trend of $x(\mathrm{M} 2)$ vs. $r$.

Finally, the respective best fits in the range of interatomic distances between 390 and $410 \AA$ are displayed in Figure 7 for all the samples. Although the absolute $G(r)$ intensity is decreased with respect to the local scale because of the instrumental contribution (see Figure 3), no noteworthy fit residual was observed. The randomly distributed difference curve accounts for some noise, at a minimum, though, thanks to the high counting statistics. The good quality of the fit further supports the goodness of our approach.

\section{CONCLUSION}

PDF analysis of CYO samples with $\mu=0.250,0.313$, 0.344 , and 0.438 composition was carried out. Several models conceiving the structural relationship between fluorite $\left(\mathrm{CeO}_{2}\right.$-like $)$ and $\mathrm{C}$-type $\left(\mathrm{Y}_{2} \mathrm{O}_{3}\right.$-like $)$ phases were evaluated through box-car refinements: (i) a two-phase model (i.e., coexisting $\mathrm{Y}_{2} \mathrm{O}_{3}$-like and $\mathrm{CeO}_{2}$-like phases), in which cell parameters and the $x(\mathrm{M} 2)$ cation coordinate were either fixed or free to refine; (ii) a single $\mathrm{C}$-type phase, refining either isotropic or anisotropic displacement parameters; and (iii) a single fluorite phase.

The model best describing the $G(r)$ curves up to high $r$, attaining both accurate fits and meaningful structural parameters, is a simple C-type phase with isotropic displacement parameters. Nanometre-sized dopant domains were determined as the range in real space in which the C-type phase best fitted the experimental $G(r)$. Gradual transformation of C-type into fluorite was observed with increasing $r$ in the $\mu=0.250,0.313$, and 0.344 samples. Different models, however, generally agree in the estimate of the C-type domain sizes, that is, nearly $100-140 \AA$ for $\mu=0.250$ and 0.313 , nearly $180-200 \AA$ for $\mu=$ 0.344 , and in excess of $500 \AA$ for $\mu=0.438$.

\section{ACKNOWLEDGEMENTS}

The authors acknowledge the European Synchrotron Radiation Facility for provision of beam time; they wish to thank Dr. A. N. Fitch $(E S R F)$ for assistance in using the ID31 beamline and also Dr. P. Masala (Università degli Studi di Milano) for useful discussions.

Billinge, S. J. L. and Levin, I. (2007). "The problem with determining atomic structure at the nanoscale," Science 316(5824), 561-565.

Coduri, M., Brunelli, M., Scavini, M., Allieta, M., Masala, P., Capogna, L., Fischer, H. E., and Ferrero, C. (2012a). "Rare Earth doped ceria: a combined X-ray and neutron pair distribution function study," Z. Kristallogr. 227, 272-279.

Coduri, M., Scavini, M., Allieta, M., Brunelli, M., and Ferrero, C. (2012b). "Local disorder in yttrium doped ceria $\left(\mathrm{Ce}_{1-x} \mathrm{Y}_{x} \mathrm{O}_{2-x / 2}\right)$ probed by joint X-ray and Neutron Powder Diffraction," J. Phys. Conf. Ser. 340, 1-10.

Coduri, M., Scavini, M., and Allieta, M. (2013a). "Defect structure of Y-doped ceria on different length scales," Chem. Mater. 25, 4278-4289.

Coduri, M., Scavini, M., Brunelli, M., and Masala, P. (2013b). "In situ pair distribution function study on lanthanum doped ceria," Phys. Chem. Chem. Phys. 15, 8495-8505.

Coduri, M., Scavini, M., Brunelli, M., Pedrazzin, E., and Masala, P. (2014). "Structural characterization of Tb- and Pr-doped ceria," Solid State Ion. 268, 150-155.

Farrow, C. L., Juhás, P., Liu, J. W., Bryndin, D., Božin, E. S., Bloch, J., Proffen, Th., and Billinge, S. J. L. (2007). "PDFfit2 and PDFgui: computer programs for studying nanostructure in crystals," J. Phys. Condens. Matter 19, 335219.

Foschini, C. R., Souza, D. P. F., Paulin Filho, P. I., and Varela, J. A. (2001). "AC impedance study of $\mathrm{Ni}, \mathrm{Fe}, \mathrm{Cu}, \mathrm{Mn}$ doped ceria stabilized zirconia ceramics," J. Eur. Ceram. Soc. 21(9), 1143-1150.

Goodenough, J. B. (2003). "Oxide-ion electrolytes," Annu. Rev. Mater. Res. 91, 128.

Gregori, G., Rahmati, B., Sigle, W., van Aken, P. A., and Maier, J. (2011). "Thermal removal of gallium from gallia-doped ceria," Solid State Ion. 192(1), 65-69.

Kim, N. and Stebbins, J. F. (2007). "Vacancy and cation distribution in yttriadoped ceria: an ${ }^{89} \mathrm{Y}$ and ${ }^{17} \mathrm{O}$ MAS NMR study," J. F. Chem. Mater. 19, $5742-5747$.

Larson, A. C. and Von Dreele, R. B. (2000). General structure analysis system (GSAS) (Report LAUR 86-748), Los Alamos, National Laboratory.

Leoni, M., Confente, T., and Scardi, P. (2006). "PM2K: a flexible program implementing Whole Powder Pattern Modelling," Z. Kristallogr. 23, 249254.

Minervini, L., Zacate, M. O., and Grimes, R. W. (1999). "Defect cluster formation in $\mathrm{M}_{2} \mathrm{O}_{3}$-doped $\mathrm{CeO}_{2}$," Solid State Ion. 116(3-4), 339-349.

Ou, D. R., Mori, T., Ye, F., Zou, J., and Drennan, J. (2006). "Microstructures and electrolytic properties of yttrium-doped ceria electrolytes: dopant concentration and grain size dependences," J. Acta Mater. 54, 3737.

Park, Y. S., Sohn, H. Y., and Butt, D. P. (2000). "Thermal removal of gallium from gallia-doped ceria,” J. Nucl. Mater. 280(3), 285-294.

Pechini, M. P. (1967). U.S. patent 3,330,697.

Qiu, X., Thompson, J. W., and Billinge, S. J. L. (2004). "PDFgetX2: a GUI-driven program to obtain the pair distribution function from X-ray powder diffraction data," J. Appl. Crystallogr. 37, 678.

Scardi, P. and Leoni, M. (2005). "Diffraction whole-pattern modelling study of antiphase domains in $\mathrm{Cu}_{3} \mathrm{Au}$," Acta Mater. 53, 5229-5239.

Scavini, M., Coduri, M., Allieta, M., Brunelli, M., and Ferrero, C. (2012). "Probing complex disorder in $\mathrm{Ce}_{1-x} \mathrm{Gd}_{x} \mathrm{O}_{2-x / 2}$ using the pair distribution function analysis," Chem. Mater. 24, 1338-1345.

Shannon, R. D. and Prewitt, C. T. (1969). "Effective ionic radii in oxides and fluorides," Acta Crystallogr. B. 25, 925-946.

Toby, B. H. (2001). "EXPGUI, a graphical user interface for GSAS," J. Appl. Crystallogr. 34, 210-213.

Yamazaki, S., Matsui, T., Ohashi, T., and Arita, Y. (2000). "Defect structures in doped $\mathrm{CeO}_{2}$ studied by using XAFS spectrometry," Solid State Ion. 913, 136. 